\title{
Derecho conductual y nudges: implicaciones jurídicas y lingüísticas
}

\section{Behavioral law and nudges: legal and lingüistic implications}

\author{
Juli Ponce Solé \\ Universidad de Barcelona (España) \\ ORCID: https://orcid.org/0000-0002-1977-5063 \\ jponce@ub.edu
}

\section{NOTA BIOGRÁFICA}

Catedrático de Derecho Administrativo. Es Doctor en Derecho por la Universidad de Barcelona, ha obtenido el Master of Arts por la Academy of European Public Law y ha realizado estancia de investigación en la Harvard Law School, donde cursó el Program of Instruction for Lawyers. Sus principales líneas de investigación giran en torno al Derecho Público y la Gestión Pública.

\section{Estrella Montolío Durán}

Universidad de Barcelona (España)

ORCID: https://orcid.org/0000-0003-4237-2200

emontoliod@ub.edu

\section{NOTA BIOGRÁFICA}

Catedrática de Lengua española de la Universitat de Barcelona. Coordinadora el grupo de investigación Estudios del Discurso Académico y Profesional (EDAP www.ub.edu/edap). Especialista en clarificación del lenguaje jurídico y administrativo. Investiga sobre los procesos comunicativos en los ámbitos profesionales. Algunas publicaciones son Hacia la modernización del discurso jurídico (ed. 2012) o El derecho a entender (con M. Tascón, 2020).

José-Andrés Rozas Valdés

Universidad de Barcelona (España)

ORCID: https://orcid.org/0000-0001-5749-7001

jarozas@ub.edu

\section{NOTA BIOGRÁFICA}

Catedrático de Derecho financiero y tributario (Universidad de Barcelona), y Vocal del Consejo para la Defensa del Contribuyente (Ministerio de Hacienda, Gobierno de España). Una de sus líneas de investigación preferentes es la gobernanza fiscal. Autor de cinco monografías, editor de otras tres, ha publicado más de doscientos originales con una marcada impronta de Derecho comparado.

\section{RESUMEN}

El objetivo del presente estudio es considerar los conocimientos sobre el comportamiento (behavioural insights), que han conducido al llamado Derecho conductual (behavioural law) y a los acicates (nudges), 
GAPP. Nueva Época - N. 25, marzo 2021 - ISSN: 1989-8991 - DOI: https://doi.org/10.24965/gapp.i25.10869 - [Págs. 58-72] Número monográfico - Los nudges y el diseño conductual de políticas públicas

Derecho conductual y nudges: implicaciones jurídicas y lingüísticas Juli Ponce Solé / Estrella Montolío Durán / José-Andrés Rozas Valdés

desde una perspectiva jurídica, imprescindible en su necesario análisis inter/transdisciplinario. Partiendo de esa relevancia jurídica de los acicates, se analizan cuestiones concretas referentes a su incidencia en los ciudadanos desde la perspectiva de un Estado Social y Democrático de Derecho como el español.

\title{
PALABRAS CLAVE
}

Derecho conductual; acicate; autoritarismo; proporcionalidad; buena administración; lenguaje administrativo.

\begin{abstract}
The aim of this study is to consider the knowledge about behavior (behavioral insights), which has led to behavioral Law and nudges, from a legal perspective, essential in its necessary inter/transdisciplinary analysis.
\end{abstract}

Based on this legal relevance of incentives, we then analyze specific issues relating to their impact on citizens from the perspective of the Rule of Law, the Social Rights and the Democracy.

\section{KEYWORDS}

Behavioral law; nudging; authoritarianism; proportionality; good administration; administrative language.

\section{SUMARIO}

1. INTRODUCCIÓN. 2. ¿SUPONE EL DERECHO CONDUCTUAL UN CAMBIO DE PARADIGMA JURÍDICO? 2.1. EJEMPLOS PRÁCTICOS DE ACICATES (NUDGES) Y SU RELACIÓN CON EL DERECHO. 2.2. EL DERECHO, LA TEORÍA DE LA ELECCIÓN RACIONAL Y EL IMPACTO DEL DERECHO CONDUCTUAL: DE LA HUMILDAD Y DE LA NECESARIA Y DIFÍCIL TRANSDISCIPLINARIEDAD. 2.3. CRÍTICAS JURÍDICAS A LOS ACICATES Y RÉPLICAS: LA INTERVENCIÓN ADMINISTRATIVA QUE DEJA LIBERTAD, EL AUTORITARISMO DEL DERECHO ADMINISTRATIVO ESPAÑOL Y EL PRINCIPIO DE PROPORCIONALIDAD (REMISIÓN). 3. DERECHO, ACICATES, LENGUAJE ADMINISTRATIVO. 3.1. PROCEDIMIENTO ADMINISTRATIVO DE TOMA DE DECISIONES ADMINISTRATIVAS: ACTOS ADMINISTRATIVOS, REGLAMENTOS, PLANES, CONTRATOS Y CONVENIOS. EL DERECHO DÉBIL (SOFT LAW). 3.2. EL LENGUAJE ADMINISTRATIVO Y LOS ACICATES: DERECHO A ENTENDER, CLARIDAD Y ENMARCADO E INFORMACIÓN INTELIGENTE (FRAMING Y SMART DISCLOSURE). LAS NOTIFICACIONES Y LAS PUBLICACIONES ADMINISTRATIVAS. 3.3 ORGANIZACIÓN ADMINISTRATIVA: LAS UNIDADES ADMINISTRATIVAS CONDUCTUALES. 3.4. TIPOS DE ACICATES DESDE UNA PERSPECTIVA JURÍDICA: INFORMALES Y FORMALES; PÚBLICOS (DIRIGIDOS A CIUDADANOS Y EMPRESAS, A DECISORES PÚBLICOS Y A EMPLEADOS PÚBLICOS) Y PRIVADOS. 4. ESTADO SOCIAL Y DEMOCRÁTICO DE DERECHO Y ACICATES. 4.1. PRINCIPIO DE LEGALIDAD, AUTONOMÍA DE LOS PRIVADOS Y PRINCIPIO DE PROPORCIONALIDAD: ¿EL GOBIERNO Y LAADMINISTRACIÓN ESTÁN OBLIGADOS JURÍDICAMENTE A UTILIZAR ACICATES? 4.2. PRINCIPIOS DE BUEN GOBIERNO Y DERECHO A UNA BUENA ADMINISTRACIÓN. 4.2.1. Acicates, principio jurídico de eficacia y cumplimiento de la legalidad: evaluación normativa y cultura de la legalidad. 4.2.2. Acicates, principio jurídico de eficiencia, principio jurídico de economía y recursos públicos. 4.2.3. Acicates y prevención de la corrupción. 4.3. ESTADO DEMOCRÁTICO Y ACICATES: TRANSPARENCIA, PUBLICIDAD ACTIVA, DERECHO DE ACCESO A LA INFORMACIÓN PÚBLICA. 4.4. ESTADO SOCIAL Y ACICATES: LOS DERECHOS SOCIALES Y ECONÓMICOS Y SU RESPETO, PROTECCIÓN Y PROMOCIÓN. 5. REFLEXIONES FINALES 6. REFERENCIAS BIBLIOGRÁFICAS.

\section{INTRODUCCIÓN ${ }^{1}$}

El objeto del trabajo es las relaciones del Derecho público, específicamente el Derecho administrativo (es decir, el que se ocupa de la actividad administrativa y abarca también a la regulación de la sociedad,

1 El presente estudio es un resultado del proyecto, del que los tres autores son miembros, RED2018-102761-T: "El nudging aplicado a la mejora de la regulación: derecho y políticas públicas conductuales", cuya página web es: Nudging aplicado a la Mejora de la Regulación - red temática de investigación transdisciplinar (wordpress.com).

Agradecemos los comentarios efectuados por los editores y evaluadores. 
Cassese, 2010, p. 284-285) y el Derecho tributario (esto es, el que regula la obtención de ingresos por parte de los entes públicos por medio de los tributos), con el Derecho conductual y los nudges, que traduciremos por acicates, de acuerdo con la expresión usada por Grande Serrano (2019).

En el marco de esas relaciones, abordaremos problemas específicos, poco tratados hasta el momento, como el impacto del principio de proporcionalidad en el uso de acicates o el juego del principio de legalidad y de la reserva de ley.

En este estudio se va a intentar responder a preguntas como las siguientes: ¿Qué implicaciones tiene el surgimiento del Derecho conductual? ¿Cómo encajan los acicates en el Derecho y cómo se ven afectados aquéllos por éste? ¿Impone el principio de proporcionalidad el uso de acicates en determinadas ocasiones? ¿Este uso puede ayudar a rebajar el autoritarismo detectado por la doctrina en el ejercicio de las potestades administrativas en España? ¿Cómo se relaciona el derecho a una buena administración con el Derecho conductual?

Las hipótesis del análisis son que el Derecho conductual, esto es, el basado en los conocimientos del comportamiento humano, puede suponer un cambio de paradigma jurídico y que el Derecho puede tener una incidencia importante en cuándo y cómo se usan los acicates, en garantía de los derechos de los ciudadanos, incluido el de buena administración, por lo que debe ser tenido muy en cuenta por el resto de las ciencias que se ocupan del tema.

El trabajo se divide en cuatro partes. Tras esta introducción, se analiza la razón por la que los conocimientos existentes del comportamiento humano pueden suponer un cambio relevante en el Derecho, al cuestionar la llamada Teoría de la Elección Racional (en adelante, TER), lo que comporta un cambio de perspectiva, que va más allá de la mera aplicación de acicates, concluyendo que dicha aplicación puede ser posible y útil para contrarrestar el autoritarismo característico del Derecho público español.

En el tercer apartado se intenta analizar en qué ámbitos propios del Derecho administrativo y tributario puede tener mayor impacto la perspectiva del Derecho conductual. A continuación, se ensaya una clasificación de acicates, en función de su formalización jurídica o de su informalidad y de quien los emplea (el sector público o el privado), si bien en el presente estudio nos ocupamos solo de los acicates desarrollados por el poder ejecutivo.

Finalmente, se considerará el encaje de los acicates en la cláusula de Estado Social y Democrático de Derecho del art. 1 de la Constitución Española (en adelante, CE), analizando relevantes cuestiones asociadas.

\section{2. ¿SUPONE EL DERECHO CONDUCTUAL UN CAMBIO DE PARADIGMA JURÍDICO?}

\subsection{Ejemplos prácticos de acicates (nudges) y su relación con el Derecho}

El artículo quinto de la Ley española 30/1979, de 27 de octubre, sobre extracción y trasplante de órganos, establece que la donación de órganos se producirá a falta de constancia expresa de la oposición del fallecido. Esto es, se utiliza en la norma una opción por defecto en favor de la donación.

Por otra parte, es frecuente encontrar en los baños de aeropuertos y otros locales, públicos y privados, un dibujo de una mosca en los urinarios masculinos, con el fin de mejorar la atención y puntería de los caballeros (Hooker, 2017).

IMAGEN 1. EJEMPLO de ACICATE EN UN BAÑo MASCULINO

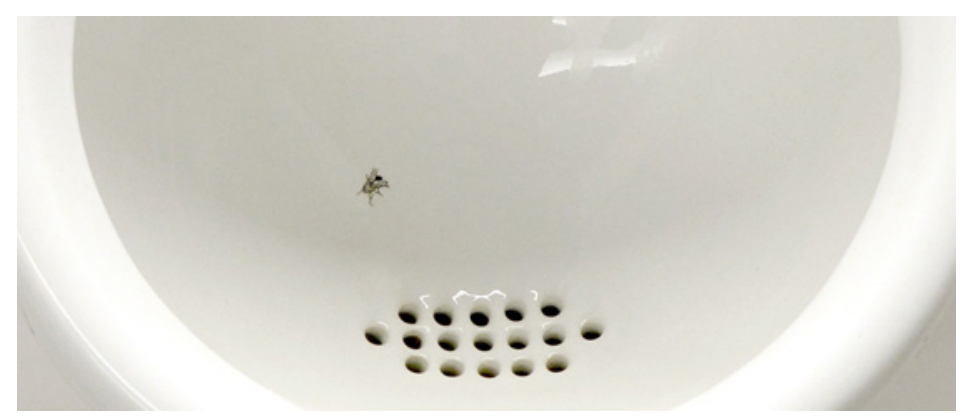

Fuente: Creative Commons, http://i.stack.imgur.com/pZ5W8.jpg. 
GAPP. Nueva Época - N. 25, marzo 2021 - ISSN: 1989-8991 - DOI: https://doi.org/10.24965/gapp.i25.10869 - [Págs. 58-72] Número monográfico - Los nudges y el diseño conductual de políticas públicas

Derecho conductual y nudges: implicaciones jurídicas y lingüísticas Juli Ponce Solé / Estrella Montolío Durán / José-Andrés Rozas Valdés

¿Qué tienen en común estos casos aparentemente tan distintos? Se trata de supuestos en que se utiliza un incentivo, un empujoncito, un incentivo, un acicate (nudge) para conseguir la modificación del comportamiento de un ciudadano (el posible donante o el posible usuario sin puntería de un baño en un aeropuerto), sin limitar ni prohibir nada y sin coste, o con un coste inapreciable, para la Administración. Todo ello con el objetivo de conseguir una finalidad de interés general, sea obtener más órganos para la donación a personas que lo necesitan para mantener o recuperar su salud, sea reducir el gasto público en limpieza.

Estamos, pues, como es sabido, ante acicates, esto es (Thaler y Sunstein, 2009):

«cualquier aspecto de la arquitectura de las decisiones que modifica la conducta de las personas de una manera predecible sin prohibir ninguna opción ni cambiar de forma significativa sus incentivos económicos. Para que se pueda considerar como nudge, debe ser barato y fácil de evitar. Los nudges no son órdenes».

Los acicates se despliegan por el poder público en virtud de lo que se ha llamado paternalismo libertario, o, en terminología que preferimos, adaptada a nuestra cultura, intervención liberal (Cassese, 2016).

Pero, como veremos, la manera de articular estos acicates puede variar notablemente.

En el primer caso, existe una formalización jurídica, una norma que prevé el acicate. En otros casos, puede acudirse al uso de información en conexión con las normas sociales y el efecto reputacional (naming and shaming). Pensemos, por ejemplo, en la publicación del «listado de morosos a Hacienda», previsto en el art. 95 bis de la Ley 58/2003, de 17 de diciembre, General Tributaria.

En el segundo caso, en cambio, no existe norma previa específica que prevea el acicate. Se trata de una mera decisión de gestión, discrecional, no formalizada, aunque no exenta de orientaciones y límites jurídicos, como veremos.

\subsection{El Derecho, la teoría de la elección racional y el impacto del Derecho conductual: de la humildad y de la necesaria y difícil transdisciplinariedad}

El Diccionario Panhispánico del Español Jurídico define el Derecho como «conjunto de principios, normas, costumbres y concepciones jurisprudenciales y de la comunidad jurídica de los que derivan las reglas de ordenación de la sociedad y de los poderes públicos, así como los derechos de los individuos y sus relaciones con aquellos» (Real Academia Española, 2020a).

Por tanto, el Derecho no son sólo normas jurídicas. El Derecho incluye principios generales del Derecho, explicitados o no en normas, y usados frecuentemente por la jurisprudencia, así como la costumbre, que es una puerta de entrada de las normas sociales al ordenamiento jurídico. El Derecho, pues, es más complejo que la mera existencia de normas jurídicas. Y, en todo caso, no debe confundirse el Derecho con la moral personal y las normas sociales, tres sistemas normativos diferentes que regulan separadamente la conducta humana, si bien delimitarlos con precisión es a veces difícil, puesto que existe una interrelación entre ellos. A veces coinciden parcialmente, a veces entran en conflicto.

En lo que se refiere específicamente al Derecho, éste «es un sistema conductual. Busca moldear el comportamiento humano-regular, incentivar, impulsar a los individuos a que se comporten de cierta manera y no de otras» (Ulen, 2015, p.25). Para ello, se ha basado tradicionalmente en la denominada Teoría de la Elección Racional (TER)².

De acuerdo con la TER, los poderes públicos que regulan comportamientos humanos -nos centramos aquí en el poder ejecutivo- lo hacen en el entendido de que las prohibiciones y limitaciones que se impongan serán cumplidas por los ciudadanos, que harán un cálculo coste-beneficio, maximizarán sus opciones y cumplirán las regulaciones ante la amenaza de los castigos diseñados normativamente.

La asunción de que quien debe cumplir la regulación es un homo economicus es la que ha regido el diseño de las regulaciones tradicionalmente; regulaciones que, además, por lo general, no han sido evaluadas ex post, tras ser promulgadas, pues si lo dice una norma jurídica que impone un castigo adecuado por su incumplimiento, el ciudadano cumplirá (se supone) con ella, al ser una persona racional que maximiza sus intereses.

Sin embargo, los estudios sobre la cultura de la legalidad ponen de relieve que en el cumplimiento o no de las normas jurídicas tienen peso distintos elementos, no sólo el cálculo racional perfecto (por ejemplo, Villoria y Jiménez, 2014, Güemes y Wences, 2019). Así, desde el ámbito de la Criminología, se propone un modelo

2 Si bien mucho antes de la formulación moderna de la teoría lo hizo ya de forma intuitiva, como demuestra la creación del modelo de hombre malo de Holmes (1897). Ese hombre malo, primo hermano del homo economicus, se caracterizaría por tratarse de un decisor que sólo se ocupa de sus propios intereses y no duda en desobedecer la ley si puede obtener beneficios para sí. 
GAPP. Nueva Época - N. 25, marzo 2021 - ISSN: 1989-8991 - DOI: https://doi.org/10.24965/gapp.i25.10869 - [Págs. 58-72] Número monográfico - Los nudges y el diseño conductual de políticas públicas

Derecho conductual y nudges: implicaciones jurídicas y lingüísticas Juli Ponce Solé / Estrella Montolío Durán / José-Andrés Rozas Valdés

de análisis de los comportamientos fraudulentos en el ámbito tributario en base a una combinación de tres factores: Willing -Be Able-Daring (WBAD), voluntad, capacidad y audacia (Elffers, 2000). Dicho de otro modo: moral, oportunidad, osadía. La incidencia de uno u otro factor varía debido a circunstancias tanto subjetivas como objetivas. Defrauda más quien menores restricciones morales tiene, disfruta de mayores oportunidades al respecto, y con mayor osadía se comporta frente al riesgo, eventual, de ser descubierto y sancionado.

A estos tres elementos habría que añadir lo que un especialista en Derecho penal denominaría error de Derecho o de prohibición. La norma tributaria se incumple, también, por desconocimiento de su contenido, o del modo adecuado en el que cabe interpretarla, pues, en demasiadas ocasiones, tiene graves defectos técnicos (volveremos luego en el epígrafe 4 sobre ello a propósito del Derecho conductual y la calidad normativa).

Pues bien, ese modelo de racionalidad de las personas que son reguladas es lo que las aportaciones de las ciencias del comportamiento discuten. ¿Puede entonces el Derecho prescindir de estas aportaciones? Creemos que no. Y ello conduce a que dichas aportaciones supongan algo más que el uso de acicates: en el Derecho (administrativo y tributario, en lo que ahora nos importa) pueden significar un cambio de paradigma, en el sentido de (Kuhn, 1996).

Un nuevo paradigma, en el que las personas reguladas pasen a ser el centro de la regulación (Cassese, 2016), en el que se abandone el formalismo jurídico propio de la cultura jurídica iberoamericana (Atienza 2006), y el Derecho se preocupe también por la efectividad real de las normas jurídicas, para corregirlas en lo necesario, a fin de modificar efectivamente el comportamiento de los ciudadanos mediante su cumplimiento real (Araguàs et al., 2017).

Un nuevo paradigma, pues, en el que el diseño normativo busque ser eficaz teniendo en cuenta cómo deciden realmente las personas a las que se dirigen las normas, no dando por supuesta una racionalidad absoluta inexistente.

Es en ese nuevo paradigma en que se insertan los acicates, como un elemento del nuevo Derecho conductual (Unión Europea, 2016, p.10). Ese Derecho conductual ha de incorporar la relevancia de la inter/ transdisciplinariedad para la resolución de problemas complejos, lo que supone, por tanto, la necesaria humildad de los juristas (Ulen, 2015, p.27) para poder entrar en un diálogo fructífero con otras especialidades. Pero en esa transdisciplinariedad, a la vez, el Derecho conductual reivindica su papel imprescindible en el concierto de las ciencias sociales (Cassese, 2012), ciencias que, por su parte, deben dejar de realizar análisis de la realidad prescindiendo del Derecho y del marco jurídico existente, como todavía sucede con frecuencia (Capo, 2005).

En definitiva, «el derecho conductual es una de los más importantes -si no el más importante- desarrollos académicos de la era moderna» (Ulen, 2015, p.57).

\subsection{Críticas jurídicas a los acicates y réplicas: la intervención administrativa que deja libertad, el autoritarismo del Derecho Administrativo español y el principio de proporcionalidad (remisión)}

Nos concentramos aquí en los reparos que parte de la doctrina jurídica ha formulado al Derecho conductual (pues ningún cambio de paradigma se efectúa sin resistencias, como es sabido).

Así, por ejemplo, se señala que los acicates provienen de un entorno jurídico muy distinto, como es el de los EE.UU. y el Reino Unido (Velasco, 2018, Carbonell, 2018). En ambos países, especialmente en el primero, los acicates buscarían legitimar la intervención pública en una cultura reacia a esta. Sin embargo, en otros países, entre ellos España, en los que la intervención pública se acepta con mayor naturalidad, estos acicates tendrían menor razón de ser o ninguna en absoluto.

Mas la utilización del Derecho conductual y de los acicates, aunque se haya impulsado más intensamente desde los países citados, sigue revistiendo interés para otros países en los que la intervención administrativa se acepta con mayor facilidad, ya que el Derecho conductual aporta instrumentos útiles para el regulador (Cassese, 2016), que, además, tienen la virtud de llamar la atención sobre quien es regulado y su cumplimiento efectivo de la regulación.

Se ha aducido también que los acicates no pueden sustituir completamente a la regulación del tipo «ordeno y mando», que establece limitaciones y prohibiciones, y penas y sanciones. Pero tal hipotética sustitución no parece haberse propuesto en ningún momento, sino que se ha señalado cómo el ordeno y mando tiene todo el sentido en ciertas ocasiones, mientras que, en otras, el uso de acicates puede ser más efectivo (sobre las posibilidades e insuficiencias de los acicates en relación con la reducción del tabaquismo y los accidentes de tráfico, Pinilla, 2017). 
GAPP. Nueva Época - N. 25, marzo 2021 - ISSN: 1989-8991 - DOI: https://doi.org/10.24965/gapp.i25.10869 - [Págs. 58-72] Número monográfico - Los nudges y el diseño conductual de políticas públicas

Derecho conductual y nudges: implicaciones jurídicas y lingüísticas Juli Ponce Solé / Estrella Montolío Durán / José-Andrés Rozas Valdés

No se trata, pues, de substitución, sino de complementariedad. Los acicates forman parte de la denominada actividad de fomento administrativa; en concreto, se trata de una actividad de fomento sofisticada y científicamente informada, un fomento no basado en incentivos económicos. Como es sabido, diversas técnicas de intervención pueden ser usadas de forma intercambiable, en función de las circunstancias (MartínRetortillo, 1988).

En tercer lugar, también se ha planteado que los acicates tendrían un papel muy marginal en el Derecho. Pero aun aceptando que ello sea cierto -lo que está por ver- ese papel, sea el que sea, si es útil para el servicio al interés general no puede ser desdeñado.

En ocasiones, se afirma también que los acicates son de efectividad dudosa. Lo cierto es que cada vez se cuenta con más estudios que intentan medir dicha eficacia, que, en todo caso, probablemente será distinta en función del tipo de acicate y del sector, y que iremos conociendo a medida que esos estudios crezcan y estén disponibles.

Ahora bien, la crítica de la falta de efectividad supone reprochar al Derecho conductual algo de lo que se presupone goza el Derecho basado en la TER. Pero ¿cómo sabemos que el Derecho basado en la TER es siempre eficaz? Sobre todo, teniendo en cuenta la habitual ausencia de evaluaciones ex post de normas jurídicas, como sabemos. ¿No será que se presume dicha eficacia por una especie de fetichismo de la ley (Duguit, 1913, p.114), de mística o idolatría, que supone que se cargue con la prueba de su efectividad a los acicates, sin exigirla por igual a las limitaciones y prohibiciones normativas?

Se afirma igualmente que los acicates, para ser efectivos, han de ser opacos, y eso implicaría el reforzamiento de una especie de manipulación de los ciudadanos (Glaeser, 2006), opacidad que encaja mal en nuestro Derecho, que promueve la transparencia. El caso es que la opacidad de los acicates no es una condición inherente a estos. Nada debe ser escondido, y todo debe ser transparente pues de hecho, la transparencia debería ser parte de una especie de Carta de Derechos para los acicates, que deben ser visibles, escudriñados y controlados (así lo afirma Sunstein, 2020, p.55). De hecho, existen ya estudios que muestran que su efectividad no decrece si son conocidos (Bruns et al., 2018).

En esa línea, se señala que los acicates serían peligrosos, porque podrían manipular a las personas, al utilizar los conocimientos relativos a su comportamiento. Aunque dicho peligro pueda existir, y deba ser controlado jurídicamente, no parece mayor que el peligro que otros tipos de incentivos, las limitaciones y prohibiciones, suponen para los derechos de los ciudadanos, lo que no hace que se descarte su uso cuando es necesario para el interés general.

En suma, junto a argumentaciones razonables, algunas críticas parecen mostrar una cierta resistencia a la innovación jurídica, resistencia que se ha señalado como un rasgo propio de nuestra cultura jurídica (Doménech, 2016).

Con todo, frente a estos obstáculos, lo cierto es que, en el contexto específico español, creemos que los acicates, como expresión del Derecho conductual, pueden permitir contrarrestar el autoritarismo. Ciertamente, el autoritarismo es un componente histórico del Derecho Administrativo en general (Cassese, 2014, p. 443-444), pero caracterizaría especialmente al Derecho administrativo español (Baño León, 2016 y 2017), lo que probablemente se explica por nuestras especiales circunstancias históricas. Ello parece llevar en España a regular en exceso ${ }^{3}$ y a una actividad administrativa excesivamente invasiva de la esfera de los ciudadanos.

Si se acepta que dicho autoritarismo del Derecho administrativo español existe, entonces el Derecho conductual, y los acicates en particular, pueden suponer un interesante corrector, en el sentido de promover modificaciones del comportamiento de los ciudadanos menos invasivas y restrictivas y más acordes con el importante principio de proporcionalidad (ver apartado 4). Operarían en sentido contrario que en EE. UU.: aquí no necesitan legitimar la intervención, sino hacerla más proporcionada.

\section{DERECHO, ACICATES, LENGUAJE ADMINISTRATIVO}

El Derecho conductual administrativo, usando conocimientos de las ciencias del comportamiento, puede tener incidencia en distintas instituciones jurídicas, como vamos a ver brevemente a continuación.

3 Sólo hay que ver la cantidad de regulaciones existentes: Mora Sanguinetti (2019) las cuantifica en alrededor de 12.000 en 2018. Ello unido a su deficiente calidad no infrecuente, como destaca la doctrina, por ejemplo en el ámbito tributario Carbajo (2016), 114-115. 
GAPP. Nueva Época - N. 25, marzo 2021 - ISSN: 1989-8991 - DOI: https://doi.org/10.24965/gapp.i25.10869 - [Págs. 58-72] Número monográfico - Los nudges y el diseño conductual de políticas públicas

Derecho conductual y nudges: implicaciones jurídicas y lingüísticas

Juli Ponce Solé / Estrella Montolío Durán / José-Andrés Rozas Valdés

\subsection{Procedimiento administrativo de toma de decisiones administrativas: actos administrativos, reglamentos, planes, contratos y convenios. El derecho débil (soft law)}

Un ámbito en el que el Derecho conductual puede desplegar efectos es el de los procedimientos de toma de decisiones administrativas formalizadas. Tanto en la elaboración de actos administrativos, como de reglamentos (y proyectos de leyes por el Gobierno ${ }^{4}$; volveremos a ello en el apartado 4, a propósito de la calidad normativa), planes y contratos o convenios, el manejo de datos propios de la ciencia del comportamiento puede ser útil para diseñar de la manera más efectiva posible la decisión que se va a adoptar en desarrollo de la política pública de que se trate.

También en el ámbito del llamado Derecho débil (soft law: guías, protocolos, instrucciones, recomendaciones..., con una vinculación jurídica limitada) el uso de conocimientos conductuales puede mejorar su utilidad.

\subsection{El lenguaje administrativo y los acicates: derecho a entender, claridad y enmarcado e información inteligente (framing y smart disclosure). Las notificaciones y las publicaciones administrativas}

Un segundo ámbito de interés es el de la comunicación de información por parte de la Administración, ya sea de manera informal (carteles, diseño de páginas web...) o de manera jurídicamente formalizada (notificaciones y publicaciones).

Como ha sido señalado «el Derecho es lenguaje. Se monta sobre palabras y esas palabras incorporan conceptos» (Martín Rebollo, 2019). Como es obvio, si la finalidad de las normas y de las comunicaciones administrativas es que se cumplan, entonces debería estar garantizado que pueden ser comprendidas por las personas. Sin embargo, es bien sabido que el lenguaje jurídico y administrativo (y en mayor proporción, si cabe, el lenguaje tributario) dista de ser fácilmente comprensible para sus destinatarios. De ahí que otros países emprendieran desde los años sesenta del siglo pasado una renovación en favor de un lenguaje claro en la comunicación entre administrados y administraciones públicas (en adelante, AA.PP).

La reivindicación de este movimiento nace como una protesta ciudadana por el derecho a entender, y es pronto recogida por las autoridades ${ }^{5}$. Es difícil que los ciudadanos confíen en una administración a la que no entienden y es complicado que, sin confianza, por ejemplo, se mantenga un sistema impositivo aceptado por la población (Strandvik, 2012). Una comunicación administrativa clara, además, aporta un ahorro del gasto público (por ejemplo, Kimble, 2012), pues evita llamadas a centros telefónicos de atención ciudadana, así como desplazamientos a oficinas presenciales para recibir aclaraciones adicionales.

En el ámbito de la Unión Europea, la renovación del discurso de las instituciones iniciado a finales del siglo pasado persigue un fin político (luchar contra la desafección ciudadana generada por el llamado eurojargon) y otro económico (ej. favorecer el laborioso proceso de traducción de las lenguas de la Unión). El movimiento better regulation, va acompañado del acrónimo KISS (Keep It Short and Simple), convertido en lema comunicativo, y tiene como brújula el libro de estilo de la Unión titulado Cómo escribir con claridad (Comisión Europea, 2015).

En España, el derecho a entender, que puede vincularse concretamente con el derecho a una buena administración, al que nos referimos más adelante (apartado 4), y con la motivación de las decisiones administrativas también, ha fundamentado los trabajos y las recomendaciones finales contenidas en un Informe de 2011 (Comisión Interministerial de Modernización del Lenguaje Jurídico).

Pero la formulación adecuada del mensaje no sólo evita indefensión por incomprensión, sino también puede ser un acicate. En este sentido, entre los sesgos cognitivos de las personas identificados, se encuentra el de enmarcado (framing) por el que las personas extraemos diferentes conclusiones de los datos disponibles en función de cómo se nos presenten. Así, para decidir si someterse o no a una cirugía, no es lo mismo que se nos diga que «De 100 pacientes que se operan, 90 están vivos cinco años después», que «De 100 pacientes que se operan, 10 mueren cinco años después». La información es la misma, pero, probablemente, en el segundo caso, decidiríamos no operarnos.

4 De acuerdo con diversos estudios empíricos disponibles, alrededor del $90 \%$ de las leyes finalmente aprobadas por el parlamento español proceden de proyectos de ley enviados por el Gobierno, Jiménez Aparicio (2004).

5 Véase, como ejemplo de este largo proceso en EE.UU, la ley Plain Writing Act (Ley de escritura clara) de octubre de 2010, aprobada por el presidente Obama, que obliga a las todas las agencias y secretarías del Gobierno a que la comunicación con los ciudadanos sea comprensible. 
GAPP. Nueva Época - N. ${ }^{\circ}$ 25, marzo 2021 - ISSN: 1989-8991 - DOI: https://doi.org/10.24965/gapp.i25.10869 - [Págs. 58-72] Número monográfico - Los nudges y el diseño conductual de políticas públicas

Derecho conductual y nudges: implicaciones jurídicas y lingüísticas

Juli Ponce Solé / Estrella Montolío Durán / José-Andrés Rozas Valdés

Las notificaciones y publicaciones de las decisiones administrativas pueden utilizar el conocimiento derivado de este sesgo humano de la comparación social: tendemos a «seguir al rebaño», por lo que hasta cierto punto las conductas deseables o no deseables pueden fomentarse atrayendo la atención pública hacia lo que los demás hacen. La regulación legal actual en España, contenida en la Ley 39/2015, de 1 de octubre, del Procedimiento Administrativo Común, no se ocupa específicamente de cómo deben redactarse las notificaciones y publicaciones, y, por tanto, permite cualquier diseño y redacción que pueda ser más efectiva en el incentivo hacia una u otra conducta del ciudadano.

\section{Notificaciones administrativas y acicates}

Existen ejemplos internacionales de notificaciones que, utilizando los conocimientos de las ciencias conductuales, han resultado más efectivas que las habituales sin ese empleo (Thaler y Sunstein, 2009, sobre reducción de consumo de energía usando emoticonos y la comparación social; los experimentos demuestran que los humanos procesamos más rápidamente una instrucción cuando va acompañada de algún elemento gráfico que cuando incluye solo texto, así Thorpe, Fize y Marlot, 1996).

Una de las escasas experiencias administrativas españolas al respecto es la del Ayuntamiento de Madrid, en relación con las sanciones por infracción de tráfico. Se trabajó en la modificación del texto de éstas, haciendo muy visible el descuento que el ciudadano obtiene si paga antes de 15 días. La evaluación de la experiencia muestra menos llamadas a los números informativos y una mayor recaudación dentro de ese período (Montolio y Tascón, 2020).

\section{Publicaciones administrativas y acicates}

En el caso de las publicaciones, ya nos hemos referido antes a la publicación de la lista de morosos a Hacienda, prevista por ley. Pero más allá de este supuesto tributario, con carácter general, el art. 45 Ley 39/2015, de 1 de octubre, permite la publicación de los actos administrativos en general, si existe un interés público para ello, y contamos ya con normativa sectorial de publicación de sanciones, en particular $^{6}$.

\subsection{Organización administrativa: las unidades administrativas conductuales}

Diversos países han creado unidades específicas para experimentar en el ámbito del comportamiento humano y asesorar a las AA.PP activas encargadas de adoptar las decisiones, como la conocida británica Behavioural Insights Team, entre otras creadas en todo el mundo (Healer, 2015).

Estas innovaciones organizativas todavía no han llegado a España, aunque sería interesante incorporarlas, bien a través del diseño de nuevas unidades administrativas, bien incorporando aportaciones del comportamiento a unidades ya existentes en el nivel estatal y autonómico, y las que puedan surgir en el nivel local, de evaluación normativa (Ponce y Villoria, 2020).

\subsection{Tipos de acicates desde una perspectiva jurídica: informales y formales; públicos (dirigidos a ciudadanos y empresas, a decisores públicos y a empleados públicos) y privados}

El Derecho conductual, pues, da lugar a la formulación de acicates formales (por ejemplo, una regulación que establece una opción por defecto, como en la donación de órganos; la previsión legal de publicación de ciertos datos, como en el caso de los morosos a Hacienda...) e informales o basados en la actividad material o técnica (puede ser el dibujo de una mosca en un urinario público, la instalación un reductor de velocidad en la vía pública, etc.).

Estos acicates informales o que implican actividad material o técnica no pueden situarse nunca al margen del Derecho. Siempre traen causa de un elemento jurídico previo (ej. un contrato administrativo que enmarca la limpieza de los baños e indica cómo debe hacerse; ej. Instrucción Técnica para la instalación de reductores de velocidad y bandas transversales de alerta en carreteras (Orden FOM/3053/2008)).

6 Así, por ejemplo, los arts. 56 y 61 de la Ley 10/2010, de 28 de abril, de prevención del blanqueo de capitales y de la financiación del terrorismo prevén la sanción de amonestación pública por la comisión de infracciones muy graves. 
GAPP. Nueva Época - N. 25, marzo 2021 - ISSN: 1989-8991 - DOI: https://doi.org/10.24965/gapp.i25.10869 - [Págs. 58-72] Número monográfico - Los nudges y el diseño conductual de políticas públicas

Derecho conductual y nudges: implicaciones jurídicas y lingüísticas Juli Ponce Solé / Estrella Montolío Durán / José-Andrés Rozas Valdés

Tanto el elemento previo como el acicate en sí (su diseño, su contenido...) están limitados y orientados por el Derecho (ej. sería ilegal un reductor que provoque accidentes o un dibujo en el urinario que viole el derecho al honor y a la propia imagen de alguien... que no sea una mosca).

Otra posible distinción entre acicates sería por su autoría. Existen acicates públicos y privados. Entre los primeros, pueden existir acicates desde los tres poderes del Estado, si bien nosotros nos concentramos en los del poder ejecutivo. Entre éstos podemos distinguir aquéllos dirigidos a los ciudadanos y empresas, para modificar su comportamiento (como los ejemplos usados al inicio), los dirigidos a los propios decisores públicos y los dirigidos en general a los altos cargos y empleados públicos, con el mismo objetivo final, modificar su conducta en aras del interés general.

También los propios privados pueden establecer acicates en relación con otros privados, aunque aquí no nos detengamos en su análisis.

\section{ESTADO SOCIAL Y DEMOCRÁTICO DE DERECHO Y ACICATES}

El art. 1 de la CE define al Estado español como un Estado Social y Democrático de Derecho.

Un Estado de Derecho implica que los acicates, deben respetar el principio de legalidad, no pueden vulnerar derechos de los ciudadanos, son controlables por la jurisdicción contenciosa (y por otras vías no judiciales, como los ombudsmen) y pueden dar lugar a responsabilidad patrimonial, si concurren todos los requisitos legales.

\subsection{Principio de legalidad, autonomía de los privados y principio de proporcionalidad: ¿El Gobierno y la Administración están obligados jurídicamente a utilizar acicates?}

El principio de legalidad implica que toda actividad administrativa tenga que ser el resultado de la existencia de una norma jurídica previa que habilite la misma, otorgando las potestades necesarias para llevarla a cabo.

Se distingue, pues, la actividad administrativa de la actividad de los privados, pues el principio de libertad que la CE (artículo 1.1) consagra autoriza a los ciudadanos a llevar a cabo todas aquellas actividades que la Ley no prohíba o cuyo ejercicio no subordine a requisitos o condiciones determinadas.

El principio de legalidad (arts. 9.3 y 103.1) impide que la Administración dicte normas sin la suficiente habilitación normativa, que deberá ser por ley, si la CE lo exige (STC 83/1984, de 24 de julio, FJ 3; un ejemplo de reserva de ley referida a derechos, art. 53.1 CE. Esta ley deberá ser en su caso orgánica, art. $81 \mathrm{CE}$ ).

Además, existiendo potestad administrativa para actuar, esta no puede ejercerse de cualquier manera. Debe, en primer lugar, ejercerse de acuerdo con la norma que la otorga y con respeto de los derechos de los ciudadanos. Pensemos en el ejemplo que hemos utilizado antes sobre el enmarcado y el uso de las notificaciones y publicaciones. Si bien hemos sostenido que entendemos que ello es posible, no se podrá hacer de cualquier modo, sino que deberá respetarse, por ejemplo, el derecho a la intimidad del interesado (art. 18 CE) y la protección de sus datos (art. 40.5 y 46 Ley 39/2015 y legislación de protección de datos).

De igual modo, el uso de opciones por defecto, como la de la donación de órganos, deberá regularse por ley si afecta a derechos (en su caso, orgánica); además, no toda opción por defecto será aceptable desde una perspectiva jurídica, pues puede afectar negativamente a derechos como el de intimidad y a la protección de datos personales, como en el caso del uso de cookies se ha puesto de relieve ${ }^{7}$.

Finalmente, desde la perspectiva del control judicial de un acicate, existen diversos principios generales del Derecho que son empleados por los jueces y tribunales.

Destaca el principio de proporcionalidad (art. 4 de la ley 40/2015, de 1 de octubre, del Sector Público). En virtud de este, toda decisión administrativa debe respetar tres filtros (si no respeta los tres sucesivamente, la decisión será desproporcionada, y, por lo tanto, ilegal).

Así, además de que el acicate busque un interés general (idoneidad) y sus beneficios superen a sus cotes (proporcionalidad strictu sensu), es crucial el llamado test de necesidad.

Para superarlo, hace falta que, al buscar ese interés general, la decisión escoja la alternativa que afecte lo menos posible los derechos de los ciudadanos y que permita lograrlo, esto es, la menos gravosa.

7 Por ello, el Reglamento de la Unión Europea de Protección de Datos, 2016/69, prohíbe ahora la opción por defecto de aceptación de cookies y exige el otorgamiento de consentimiento expreso. Sobre esta cuestión, puede consultarse la Sentencia del Tribunal de Justicia de la Unión Europea de 1 de octubre de 2019, comentada en PONCE (2020b). 
GAPP. Nueva Época - N.o 25, marzo 2021 - ISSN: 1989-8991 - DOI: https://doi.org/10.24965/gapp.i25.10869 - [Págs. 58-72] Número monográfico - Los nudges y el diseño conductual de políticas públicas

Derecho conductual y nudges: implicaciones jurídicas y lingüísticas Juli Ponce Solé / Estrella Montolío Durán / José-Andrés Rozas Valdés

Este subprincipio implica que el decisor deba considerar diversas alternativas, puesto que ha de ponderar los elementos relevantes para la toma de la decisión (como exige su obligación de buena administración a que luego nos referiremos) para elegir la menos gravosa para el ciudadano. El ordenamiento jurídico en ocasiones explícita esta obligación derivada del derecho a una buena administración y del principio de proporcionalidad ${ }^{8}$.

Pues bien, si los acicates son menos invasivos de la libertad original de los ciudadanos que las limitaciones o prohibiciones regulatorias y son, al menos, igual de efectivos, entonces el principio de proporcionalidad impondría el uso de acicates ${ }^{9}$.

Y es aquí precisamente donde existe un concepto puente (Schmidt-Assman, 2006), el de eficacia, que permite conectar al Derecho con otras disciplinas (Sociología, Ciencia Política, Economía...), pues, ¿cómo podría saber un decisor administrativo la efectividad de una u otra medida a adoptar si no es basándose en la evidencia disponible?

En definitiva, volvemos a sugerir aquí que los acicates pueden ser una herramienta para contrarrestar el autoritarismo que caracterizaría al ejercicio del poder administrativo en España, como aliados del principio de proporcionalidad. Si existe algún acicate disponible, el decisor debería, cuanto menos, considerar su uso para decidir con la diligencia debida propia de su obligación de buena administración, motivar su no elección y evitar la desproporción.

\subsection{Principios de buen gobierno y derecho a una buena administración}

\subsubsection{Acicates, principio jurídico de eficacia y cumplimiento de la legalidad: evaluación normativa y cultura de la legalidad}

Los acicates y el Derecho conductual informado por las ciencias del comportamiento pueden ser una herramienta en favor del cumplimiento de los principios jurídicos de buen gobierno (ley estatal 19/2013, arts. 25 y ss. y equivalentes legislaciones autonómicas) y de la efectividad de los principios y del derecho de las personas a la buena administración, previsto en el art. 41 de la Carta de Derechos Fundamentales de la Unión Europea, implícitamente en la CE en diversa normativa y utilizado abundantemente por la jurisprudencia (Ponce y Villoria, 2020).

De acuerdo con el Tribunal Supremo español, los ciudadanos, gracias a este derecho, podemos exigirle a nuestras AA.PP «una conducta lo suficientemente diligente como para evitar definitivamente las posibles disfunciones derivadas de su actuación» (Sentencia de 18 de diciembre de 2019, entre muchas otras). En su sentencia de 15 de octubre de 2020, el Tribunal Supremo español ha subrayado que (cursivas nuestras):

«Es sabido que el principio de buena administración está implícito en nuestra Constitución (artículos 9.3, 103 y 106), ha sido positivizado en la Carta de Derechos Fundamentales de la Unión Europea (artículos 41 y 42)».

«constituye, según la mejor doctrina, un nuevo paradigma del Derecho del siglo XXI referido a un modo de actuación pública que excluye la gestión negligente».

«y -como esta misma Sala ha señalado en anteriores ocasiones- no consiste en una pura fórmula vacía de contenido, sino que se impone a las Administraciones Públicas, de suerte que el conjunto de derechos que de aquel principio derivan (audiencia, resolución en plazo, motivación, tratamiento eficaz y equitativo de los asuntos, buena fe) tiene-debe tener-plasmación efectiva y lleva aparejado, por ello, un correlativo elenco de deberes plenamente exigible por el ciudadano a los órganos públicos».

El principio jurídico constitucional de eficacia (art. 103.1 CE) integra el conjunto de principios de buena administración. El Derecho conductual y los acicates pueden contribuir a que las decisiones administrativas

8 En el ámbito de la aprobación de normas, véase el Art. 133 de la ley 39/2015, declarado constitucional por la Sentencia del Tribunal Constitucional 55/2018, de 24 de mayo, y en idéntico sentido, art. 26 de la Ley de Gobierno de 1997, modificada por la ley 40/2015. Las alternativas deben incluir una solución no normativa.

9 Este efecto de la proporcionalidad ha sido notado por algún autor jurídico, que, sin embargo, ha discutido que haya una obligación jurídica de emplear acicates porque éstos no van a ser igual de efectivos que limitaciones o prohibiciones (Schweizer, 2016).

Sin embargo, esta conclusión, en abstracto y general, no puede ser aceptable sin más en nuestra opinión. Esta presunción de eficacia en favor del modelo de ordeno y mando y de ineficacia del Derecho conductual y de los acicates es una opinión subjetiva discutible. Habrá que estar al caso concreto de cada intervención administrativa y a los datos disponibles sobre la efectividad de las distintas medidas para poder afirmar cuál sea más efectiva en cada contexto. Esa particularización de las circunstancias en la toma de cada decisión es una exigencia del derecho a una buena administración de los ciudadanos. 
GAPP. Nueva Época - N.o 25, marzo 2021 - ISSN: 1989-8991 - DOI: https://doi.org/10.24965/gapp.i25.10869 - [Págs. 58-72] Número monográfico - Los nudges y el diseño conductual de políticas públicas

Derecho conductual y nudges: implicaciones jurídicas y lingüísticas

Juli Ponce Solé / Estrella Montolío Durán / José-Andrés Rozas Valdés

sean más efectivas, esto es, que se diseñen mejor para para alcanzar los efectos deseados. Ello incluye también a las normas que provienen del poder ejecutivo, pues la calidad de la regulación, que engarza con el movimiento internacional de la better regulation, persigue sobre todo la eficacia de la norma, es decir, que efectivamente modifique el comportamiento de los ciudadanos (Xanthaki, 2011).

Ello hace que tanto la evaluación de impacto normativo ex ante como la evaluación ex post deban procurar y constatar, respectivamente, dicha eficacia, esto es, el cumplimiento normativo, el cual no depende sólo, como ya dijimos del castigo que prevea la norma, sino de otros factores a tener en cuenta.

En particular, en el ámbito del Derecho tributario, reducir su incumplimiento (del que ya hablamos en el apartado 2) pasa por rebajar su incertidumbre, y ello exige una mejora de la calidad normativa ${ }^{10}$.

Un Derecho tributario más sencillo en su configuración, más simple y automático en su aplicación (con sistemas de gestión de su cumplimiento o compliance), más previsible en las consecuencias de su incumplimiento (con una eficaz lucha contra el fraude fiscal o enforcement) y que usara acicates en la comunicación efectiva de lo que comportan e implican las políticas financieras públicas, para reforzar la moral del contribuyente respecto del cumplimiento puntual de sus obligaciones tributarias (acompañada de la participación, transparencia y ejemplaridad en la aplicación de los gastos públicos) conduciría a un incremento exponencial de su cumplimiento voluntario por los contribuyentes. En todos estos aspectos, las ciencias del comportamiento tienen mucho que aportar al Derecho tributario.

\subsubsection{Acicates, principio jurídico de eficiencia, principio jurídico de economía y recursos públicos}

Asimismo, otros principios generales del Derecho propios de la buena administración contemplados en la CE son los de eficiencia y economía en el uso de recursos públicos (art. 31.2 CE $^{11}$, Vaquer, 2011). Los acicates, dado su nulo o inapreciable coste, pueden ser instrumentos de cumplimiento de dichos principios de buena administración.

\subsubsection{Acicates y prevención de la corrupción}

Los acicates también pueden ser una herramienta para prevenir la mala administración, sea negligente o dolosa (corrupción), puesto que los decisores públicos y en general los empleados públicos, como humanos que son, no están exentos de sesgos. En un reciente informe de la OCDE (2018) se exponen una serie de medidas que pueden ayudar a prevenir la corrupción, como, por ejemplo, una opción por defecto, por la que en el inicio de todo nuevo proyecto cada empleado público involucrado reciba automáticamente un documento para declarar cualquier conflicto de interés posible.

\subsection{Estado Democrático y acicates: transparencia, publicidad activa, derecho de acceso a la información pública}

La transparencia tiene una fuerte conexión con la participación ciudadana, formando el concepto de gobierno abierto vinculado a la cláusula de Estado Democrático del art. 1.1 CE. Una nueva generación de leyes de transparencia, acceso a la información y buen gobierno desde 2013 (la estatal 19/2013, y las autonómicas posteriores) han impulsado la obligación jurídica de publicidad activa por parte de las AA.PP.

Ésta puede pasar, así, de ser un simple, pero relevante, elemento de transparencia a ser algo más: una política pública en sí misma, que busca orientar la actividad del sector privado para la realización de actividades de interés general, en función de qué información se publicita y, sobre todo, de cómo se publicita $^{12}$. Eso conlleva también la relevancia de cómo es presentada la información y el lenguaje utilizado (smart disclosure).

10 Por ejemplo, en Derecho tributario español no se contempla franquicia o umbral alguno en la sujeción a gravamen de las donaciones, de modo que, en puridad, los regalos y liberalidades de uso con motivo de onomásticas y otras celebraciones dan lugar al devengo del impuesto, siendo así que, con carácter general, el impuesto sobre donaciones viene siendo defraudado, sencillamente, por desconocimiento y por una deficiente técnica legislativa en la configuración del hecho imponible (Anderson, M. y Rozas, J. A., 2013).

11 «El gasto público realizará una asignación equitativa de los recursos públicos, y su programación y ejecución responderán a los criterios de eficiencia y economía».

12 Art. 5.5 Ley 19/2013: «Toda la información será comprensible, de acceso fácil y gratuito y estará a disposición de las personas con discapacidad en una modalidad suministrada por medios o en formatos adecuados de manera que resulten accesibles y comprensibles, conforme al principio de accesibilidad universal y diseño para todos». 
GAPP. Nueva Época - N. ${ }^{\circ}$ 25, marzo 2021 - ISSN: 1989-8991 - DOI: https://doi.org/10.24965/gapp.i25.10869 - [Págs. 58-72] Número monográfico - Los nudges y el diseño conductual de políticas públicas

Derecho conductual y nudges: implicaciones jurídicas y lingüísticas Juli Ponce Solé / Estrella Montolío Durán / José-Andrés Rozas Valdés

\subsection{Estado social y acicates: los derechos sociales y económicos y su respeto, protección y promoción}

Es conocida la conexión de los derechos sociales y económicos con la cláusula del Estado Social. El capítulo III del Título Primero de la CE (y varios Estatutos de Autonomía) establecen auténticos derechos subjetivos (salud, medio ambiente...), que despliegan obligaciones jurídicas, cuanto menos de medios, exigibles a las AA.PP, de respetarlos, protegerlos y satisfacerlos (promover es una expresión que es frecuentemente usada en los preceptos citados) de acuerdo con la clasificación clásica tripartita de obligaciones jurídicas respecto a derechos sociales (ONU, 2009).

En esa incentivación de los derechos sociales los acicates pueden ser de especial utilidad para modificar el comportamiento de los ciudadanos y dirigirlo hacia la eficacia de dichos derechos, sin necesidad de gasto público. Pensemos, por ejemplo, sobre el derecho al medio ambiente adecuado, donde determinados acicates pueden ayudar a su logro (Caridad, Fortes e Isabel, 2019), o el derecho a pensiones adecuadas, donde otros acicates pueden contribuir al mismo, al potenciar el ahorro privado, por ejemplo (Ponce, 2020a).

\section{REFLEXIONES FINALES}

La Unión Europea (2017) ha destacado como los acicates son «una herramienta especialmente interesante para responder a determinados desafíos sociales, medioambientales y económicos».

Ahora bien, como también se indica, «no deben subestimarse los riesgos y limitaciones asociados a su uso», entre ellos «la cuestión de quién decide el objetivo perseguido, es decir, lo que es deseable para la persona o la sociedad. Si se trata de un responsable público, puede orientar este objetivo, así como el nudge de manera oportunista; pero también, de forma menos intencionada, por falta de información, por ejemplo».

Entre esos riesgos surge, como ya dijimos, el de una posible manipulación, el cual se puede verse agravado por el uso de la inteligencia artificial, como ha puesto de relieve la doctrina, creando el concepto de hypernudging (Yeung, 2016) y el Consejo de Europa en su Declaración sobre las capacidades manipulativas de los procesos algorítmicos, de 13 de febrero de 2019 (Consejo de Europa, 2019).

Pero este tema ya deberá ser objeto de otros análisis futuros específicos. En todo caso, tanto las oportunidades que abren los acicates como sus límites y problemas no podrán ser tratados adecuadamente sin acudir también a la Ciencia Jurídica.

\section{REFERENCIAS BIBLIOGRAFÍCAS}

Anderson, M. y Rozas, J. A. (2013). Captaciones públicas de donaciones y donaciones benéficas: régimen civil y tributario. En J. A. Valle Zayas, J. A. Pérez Ribarés y J. R. Salelles (dirs.), Estudios sobre Derecho de la empresa en el Código civil de Cataluña, (pp. 231-251). Bosch Editores.

Atienza, M. (2006). Prólogo. En Courtis C. (coord.), Observar la Ley: ensayos sobre metodología de la investigación jurídica (pp. 10 y 11). Trotta.

Baño León, J. M. (2017). Los excesos de la llamada autotutela administrativa. En J. C. Laguna de Paz, I. Sanz Rubiales e I. de los Mozos Touya (coords.), Derecho administrativo e integración europea: estudios en homenaje al profesor José Luis Martínez López-Muñiz. Tomo II: El actuar de la Administración Pública y garantías de su efectiva legalidad, (pp. 825-842). Reus.

Baño León, J. M. (5 y 6 de febrero de 2016). El recurso administrativo como ejemplo de la inercia autoritaria del derecho público español. En F. Lopez Ramón (coord.). Las vías administrativas de recurso a debate. En Actas del XI Congreso de la Asociación Española de Profesores de Derecho Administrativo. (pp. 496-518). Instituto Nacional de Administración Pública. http://www.aepda.es/AEPDAEntrada-1181-XI-CONGRESO-DE-LA-AEPDA.aspx

Becker, G. (1968). Crime and Punishment: An Economic Approach. Journal of Political Economy, 76(2),169-217. https:// doi.org/10.1007/978-1-349-62853-7_2

Bernstein, J. H. (2015). Transdisciplinarity: A review of its origins, development, and current issues. Journal of Research Practice, 11(1). http://jrp.icaap.org/index.php/jrp/article/view/510/412

Bruns, H., Kantorowicz-Reznichenko, E., Klement, K., Jonsson, M. L. y Rahali, B. (2018). Can nudges be transparent and yet effective? Journal of Economic Psychology, 65, 41-59. https://doi.org/10.1016/j.joep.2018.02.002

Capo Giol, J. (2005). El Derecho y la Ciencia Política. En A. Castellà (coord.), La Constitución y el Ordenamiento Jurídico (pp. 464). Atelier-UB. 
GAPP. Nueva Época - N. ${ }^{\circ}$ 25, marzo 2021 - ISSN: 1989-8991 - DOI: https://doi.org/10.24965/gapp.i25.10869 - [Págs. 58-72] Número monográfico - Los nudges y el diseño conductual de políticas públicas

Derecho conductual y nudges: implicaciones jurídicas y lingüísticas Juli Ponce Solé / Estrella Montolío Durán / José-Andrés Rozas Valdés

Carbajo Vasco, D. (2016). La lucha contra la planificación fiscal agresiva y las obligaciones de información tributaria. Crónica Tributaria, (158), 109-128.

Carbonell, E. (2018). Integración de nudges en las políticas ambientales. Revista Aragonesa de Administración Pública, (Monografía XIX), (Ejemplar dedicado a: Mecanismos económicos y de mercado para la protección ambiental), 451-485. http://bibliotecavirtual.aragon.es/bva/i18n/catalogo_imagenes/grupo.cmd?path=3715369

Caridad, I., Fortes González, M. e Isabel, A. (2019). En R. Rivero Ortega y M. Cerezo Prieto (coords.), Innovación en las normas ambientales. Tirant Lo Blanch.

Cassese, S. (2012). New Paths for Administrative Law: A Manifesto. International Journal of Constitutional Law, 10 (3), 603-613 (edición en español en: http://es.globallawpress.org/wp-content/uploads/Nuevas-vi\%CC\%81aspara-el-Derecho-Administrativo.-Un-manifiesto.-Traduccio\%CC\%81n-del-ingle\%CC\%81s.-Estracto-dellibro-_Derecho-Administrativo.-Historia-y-futuro_-coleccio\%CC\%81n-blanca-.pdf). https://doi.org/10.1093/icon/ $\operatorname{mos} 038$

Cassese, S. (2010). El estado presente del Derecho Administrativo Italiano. Revista de Administración Pública, (183) septiembre-diciembre, 283-293. http://www.cepc.gob.es/publicaciones/revistas/revistaselectronicas?IDR=1\&IDN= 1265\&IDA=35646

Cassese, S. (2014). Derecho Administrativo: Historia y Futuro. INAP-Global Law Press.

Cassese, S. (2016). Exploring the legitimacy of Nudging. En A. Kemmerer, C. Möllers, M. Steinbeis y G. Wagner (eds.), Choice Architecture in Democracies. Exploring the Legitimacy of Nudging, (pp. 241-246). Hart Publishing, Nomos. https://doi.org/10.5771/9783845263939-241

Comisión Europea (2015). Cómo escribir con claridad. En Dirección General de Traducción (Comisión Europea), Publicaciones oficiales de la unión europea. https://doi.org/10.2782/259493

Consejo de Europa (14 de febrero de 2019). Declaration by the Committee of Ministers on the manipulative capabilities of algorithmic processes [Adoptada por el Comité de Ministros el 13 de febrero de 2019 en la reunión de delegados de ministros 1337]. Consejo de Europa. https://search.coe.int/cm/pages/result_details. aspx?Objectld=090000168092dd4b

Dictamen del Comité Económico y Social Europeo sobre «Integrar los nudges en las políticas europeas» (Dictamen de iniciativa) (10 de marzo de 2017). Diario oficial de la Unión Europea (2017/C 075/05 , pp. 28-32). https://eur-lex. europa.eu/legal-content/ES/TXT/PDF/?uri=CELEX:52016IE1333\&from=NL

Doménech Pascual, G. (2016). Que innoven ellos. Por qué la ciencia jurídica española es tan poco original, creativa e innovadora. InDret: Revista para el Análisis del Dereho, (2). https://indret.com/wp-content/themes/indret/ pdf/1228_es.pdf

Duguit, L. (1913). Las transformaciones del Derecho Público (traducción y estudio preliminar de Adolfo Posada y Ramón Jaén). Francisco Beltrán.

Elffers, H. (2000). But taxpayers do cooperate!. En M. Van Vugt, M. Snyder, T. R., Tyler y A. Biel (eds), Cooperation in modern society: Promoting the welfare of communities, states, and organizations (pp.184-194). Routledge. https:// doi.org/10.4324/9780203354254

Foster, N. (2008). The Journal of Comparative Law A New Scholarly Resource. The Journal of Comparative Law, (1), 1-12. https://core.ac.uk/download/pdf/2787604.pdf. https://eprints.soas.ac.uk/4408/

García de Enterria, E. (1974). Dos estudios sobre la usucapión en Derecho Administrativo. Civitas.

Giner, S., Lamo de Espinosa, E. y Torres, C. (1998). Diccionario de Sociología. Alianza editorial.

Glaeser, E. (2006). Paternalism and Psychology. The University of Chicago Law Review, 73(1), 133-156. https://doi. org/10.3386/w11789

Grande Serrano, P. (2019). La psicología económica como herramienta para incentivar el cumplimiento voluntario de las obligaciones tributarias. Revista de Contabilidad y Tributación, (439), 5-36.

Güemes, C. y Wences, I. (2019). Comportamientos incumplidores: contextos y posibles tratamientos políticos. Papers. Revista de Sociología, 104(1), 75-99. http://dx.doi.org/10.5565/rev/papers.2482

Healer, D. (2015). The Nudge Unit: How small changes can make a big difference. Penguin Random House.

Hooker, L. (2017). Have you been nudged? British Broadcasting Corporation (BBC) News. https://www.bbc.com/news/ business-41549533

Holmes, O. W. (1897). The Path of the Law. Harvard Law Review, 10(8), 457-478. https://doi.org/10.2307/1322028

Jiménez Aparicio, E. (2004). El procedimiento de elaboración de los anteproyectos de ley: la fase gubernamental. En A. Menéndez (dir.), A. Pau (dir. adjunto), La proliferación legislativa: una desafío para el Estado de Derecho (pp. 294-373). Civitas-Colegio Libre de Eméritos.

Jordano de Pozas, L. (1949). Ensayo de una teoría de fomento en el Derecho Administrativo. Revista de Estudios Políticos, (48), 41-54. http://www.cepc.gob.es/publicaciones/revistas/revistaselectronicas? IDR=3\&IDN=445\&I $\mathrm{DA}=7421$

Kimble, J. (2012). Writing for Dollars, Writing to Please. The case of Plain Language in Business Government, and Law. Carolina Academic Press.

Kunn, T. S. (1996). The Structure of Scientific Revolutions (3. ${ }^{\text {a }}$ ed.). University of Chicago.

Martín Rebollo, L. (2019). Manual de las Leyes Administrativas (3. ${ }^{a}$ ed.). Thomson Reuters Aranzadi. 
GAPP. Nueva Época - N. ${ }^{\circ}$ 25, marzo 2021 - ISSN: 1989-8991 - DOI: https://doi.org/10.24965/gapp.i25.10869 - [Págs. 58-72] Número monográfico - Los nudges y el diseño conductual de políticas públicas

Derecho conductual y nudges: implicaciones jurídicas y lingüísticas Juli Ponce Solé / Estrella Montolío Durán / José-Andrés Rozas Valdés

Martín-Retortillo, S. (1988). Derecho Administrativo Económico I. La Ley.

Montolío, E. y Tascón, M. (2020). El derecho a entender. La comunicación clara, la mejor defensa de la ciudadanía. Catarata.

Mora-Sanguinetti, J. (2019). La «complejidad» de la regulación española ¿cómo medirla? ¿qué impacto económico tiene? Información Comercial Española, ICE: Revista de economía, (907) (Ejemplar dedicado a: La mejora de la regulación), 145-162. https://doi.org/10.32796/ice.2019.907.6784

OECD (2018). Behavioural Insights for Public Integrity. Harnessing the Human Factor to Counter Corruption. OECD Publishing (OECD Public Governance Reviews). https://dx.doi.org/10.1787/9789264297067-6-en

Oficina del Alto Comisionado de las Naciones Unidas para los Derechos Humanos (2009). Preguntas frecuentes sobre los Derechos Económicos, Sociales y Culturales [Folleto Informativo número 33]. https://www.ohchr.org/ documents/publications/fs33_sp.pdf

Pérez Moreno, A. (2000). Procedimientos de conciliación, mediación y arbitraje como medios alternativos a la vía de recurso. Revista Andaluza de Administración Pública, (37), 11-37.

Pinilla, J. (octubre de 2017). ¿Cómo legislar para promover la salud pública? Los casos del tabaco y los accidentes de tráfico. Observatorio Social de La Caixa. https://observatoriosociallacaixa.org/-/como-legislar-para-promover-lasalud-publica_los-casos-del-tabaco-y-los-accidentes-de-trafico

Ponce Solé, J. y Villoria Mendieta, M. (dirs.) (2020). Anuario del Buen Gobierno y de la Calidad de la Regulación 2019. Fundación Democracia y Gobierno Local.

Ponce Solé, J. (2020a). Legislar mejor para una jubilación mejor mediante la aplicación de las ciencias conductuales: better regulation, behavioral law and economics y nudging. I Edición Premios Longevia BBV. Resolviendo los retos del envejecimiento poblacional en España, 12-70. BBVA. https://www.jubilaciondefuturo.es/recursos/doc/ pensiones/20180910/fondo-documental/premios-longevia-i.pdf

Ponce Solé, J. (2020b). El derecho a una buena administración y la personalización de los servicios públicos. Sesgos, nudging e inteligencia artificial. En B. Puentes y A. Quintiá (dirs.), El derecho ante la transformación digital. Oportunidades, riesgos y garantías (pp. 51-72). Atelier.

Ponce Solé, J. (2018). Derecho administrativo, ciencias conductuales y nudging en la gestión pública. Revista Vasca de Gestión de Personas y Organizaciones Públicas, (15), 8-27. https://www.euskadi.eus/t59auUdaWar/t59aMostra rFicheroServlet?R01 HNoPortal=true\&t59aldRevista=3\&t59aTipoEjemplar=R\&t59aSeccion=51\&t59aContenido=1\&

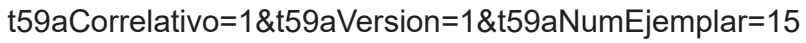

Araguàs Galcerà, I., Capdeferro Villagrasa, O., Cerrillo i Martínez, A., De Benedetto, M., Lucidi, G., Fernández, T. R., Ortí Ferrer, P., Ponce Solé, J., Ranchordas, S. y Vaquer Caballería, M. (2017). En Ponce Solé, J. y Cerrillo i Martínez, A. (coords.), Innovación en el ámbito del buen gobierno regulatorio: ciencias del comportamiento, transparencia y prevención de la corrupción. Instituto Nacional de Administración Pública (Colección Monografías). https://www.inap.es/documents/10136/1702085/PagInicia-Innovacion-en-el-ambito-del-buen-gobierno-regulatorio. pdf/a12c3a73-4551-9ba9-7d94-1a33e0e5df6b

Real Academia Española (2020a). Diccionario Panhispánico del Español Jurídico. https://dpej.rae.es/

Real Academia Española (2020b). Diccionario de la Lengua Española. https://dle.rae.es/

Real Academia Española (2020c). Diccionario del Español Jurídico. https://www.rae.es/obras-academicas/diccionarios/ diccionario-del-espanol-juridico

Rivero Ortega, R. (2012). La necesaria innovación en las instituciones administrativas. Organización, procedimiento, función pública, contratos administrativos y regulación. Instituto Nacional de Administración Pública.

Romano, S. (1964). Fragmentos de un diccionario jurídico. Ediciones Jurídicas Europa-América.

Schmidt-Assman, E. (2006). El método de la ciencia del Derecho Administrativo. En J. Barnes (ed.), Innovación y Reforma en el Derecho Administrativo (pp.15-69). Global Law Press.

Schweizer, M. (2016). Nudging and the Principle of Proportionality: Obliged to Nudge? En K. Mathis y A. Tor (eds.), Nudging: Possibilities, Limitations and Applications in European Law and Economics (pp. 93-119). Springer. https:// doi.org/10.1007/978-3-319-29562-6_7

Strandvik, I. (2012). La modernización dēl lenguaje jurídico en Suecia: ¿enseñanzas aplicables a otras tradiciones? En E. Montolío (ed.), Hacia la modernización del lenguaje jurídico. Publicacions de la Universitat de Barcelona.

Sunstein, C. R. (2020). Behavioral Science and Public Policy (Serie: Elements in Public Economics). Cambridge University Press. https://doi.org/10.1017/9781108973144

Thaler, R. H. y Sunstein, C. R. (2009). Un pequeño empujón (nudge): El impulso que necesitas para tomar las mejores decisiones en salud, dinero y felicidad. Taurus.

Thorpe, S., Fize, D. y Marlot, C. (1996). Speed of processing in the human visual system. Nature, 381(6.582), 520-522. https://doi.org/10.1038/381520a0

Ulen, T. S. (2015). La importancia del derecho conductual. Revista de Teoría del Derecho de la Universidad de Palermo, año II(1), 25-65. https://www.palermo.edu/derecho/pdf/teoria-del-derecho/n3/TeoriaDerecho_02.pdf

Sousa Lourenco, J., Ciriolo, E., Rafael Rodrigues Vieira de Almeida, S. y Troussard, X. (2016). Behavioural Insights Applied to Policy-European Report 2016. Publicaciones Oficiales de la Unión Europea. https://doi.org/10.2760/903938

Vaquer Caballeria, M. (2011). El criterio de la eficiencia. Revista de Administración Pública, (186), 91-135. http://www. cepc.gob.es/publicaciones/revistas/revistaselectronicas?IDR=1\&IDN=1282\&IDA=36259 
GAPP. Nueva Época - N. 25, marzo 2021 - ISSN: 1989-8991 - DOI: https://doi.org/10.24965/gapp.i25.10869 - [Págs. 58-72] Número monográfico - Los nudges y el diseño conductual de políticas públicas

Derecho conductual y nudges: implicaciones jurídicas y lingüísticas Juli Ponce Solé / Estrella Montolío Durán / José-Andrés Rozas Valdés

Velasco Caballero, F. (2018). «Nudges» y Derecho administrativo. Blog de Francisco Velasco [blog]. https:// franciscovelascocaballeroblog.wordpress.com/2018/05/28/nudges-y-derecho-administrativo/

Villoria, M. y Jiménez, F. (2014). Estado de Derecho, cultura de la legalidad, y buena gobernanza. En I. Wences, R. Conde y A. Bonilla (eds.), Cultura de la legalidad en Iberoamérica: desafíos y experiencias (pp. 83-118). Flacso. https://www.flacso.org/sites/default/files/Documentos/libros/secretaria-general/Cultura $\% 20 \mathrm{de} \% 20 l a \% 20$ Legalidad.pdf

Xanthaki, H. (2011). Quality of legislation: an achievable universal concept or a utopian pursuit? Quality of Legislation. Principles and Instruments: Proceedings of the Ninth Congress of the International Association of Legislation (IAL). (pp. 75-85). Nomos. https://sas-space.sas.ac.uk/4854/

Yeung, K. (2016). Hypernudge': Big Data as a mode of regulation by design. Information Communication and Society, 20(1), 118-136. https://doi.org/10.1080/1369118X.2016.1186713 